\title{
Association of Methylenetetrahydrofolate Reductase C677T Gene Polymorphisms with Mild Cognitive Impairment Susceptibility: A Systematic Review and Meta-Analysis
}

\author{
Jiahui Sun $\mathbb{D},{ }^{1,2}$ Xuefan Jiang $\mathbb{D},{ }^{1,2}$ Ming Zhao, ${ }^{1,2}$ Lina Ma, ${ }^{2}$ Hui Pei, ${ }^{2}$ Nanyang Liu $\mathbb{D},{ }^{2}$ \\ and Hao Li iD $^{2,3}$ \\ ${ }^{1}$ Graduate School, Beijing University of Chinese Medicine, Beijing, China \\ ${ }^{2}$ Department of Geratology, Xiyuan Hospital, China Academy of Chinese Medical Sciences, Beijing, China \\ ${ }^{3}$ Wangjing Hospital, China Academy of Chinese Medical Sciences, Beijing, China \\ Correspondence should be addressed to Nanyang Liu; xyhp2021@yeah.net and Hao Li; xyhplihao1965@126.com
}

Received 4 July 2021; Accepted 26 August 2021; Published 18 September 2021

Academic Editor: Gianluca Coppola

Copyright (C) 2021 Jiahui Sun et al. This is an open access article distributed under the Creative Commons Attribution License, which permits unrestricted use, distribution, and reproduction in any medium, provided the original work is properly cited.

\begin{abstract}
Background. Methylenetetrahydrofolate reductase (MTHFR) C677T (rs1801133) gene polymorphisms are related to a growing risk of Alzheimer's disease; however, whether this association applies to mild cognitive impairment (MCI) remains unclear. Objective. We conducted this meta-analysis to evaluate the contribution of MTHFR C677T (rs1801133) gene variants to the risk of MCI. Methods. PubMed, Embase, Web of Science, and China National Knowledge Infrastructure databases were searched from their inception to March 21, 2021, with language restricted to English or Chinese. We used fixed or random effects to examine the association between MTHFR C677T (rs1801133) gene variants and MCI susceptibility. Forest plots of pooled odds ratios (ORs) and 95\% confidence intervals (CIs) were generated. Results. Eight articles with 2,175 participants were included in the present meta-analysis. There was no significant association between MTHFR C677T (rs1801133) gene variants and MCI susceptibility under the allelic (OR, 1.318; 95\% CI, 0.964-1.801; $p=0.084$ ), dominant (OR, 1.296; 95\% CI, 0.925-1.817; $p=0.132$ ), recessive (OR, 1.397; 95\% CI, 0.845-2.312; $p=0.193$ ), heterozygous (OR, 1.031; 95\% CI, 0.855-1.243; $p=0.749$ ), or homozygous (OR, 1.506; 95\% CI, 0.850-2.667; $p=0.160$ ) models. Conclusion. The results suggest that MTHFR C677T (rs1801133) gene polymorphisms are not associated with MCI susceptibility. However, large-scale studies covering various factors are required.
\end{abstract}

\section{Introduction}

Mild cognitive impairment (MCI) is defined as a transitional stage between normal aging and dementia [1], that is, the symptomatic predementia stage, which does not fulfill the criterion for dementia diagnosis [2]. In the United States, age-related cognitive decline affects approximately $20 \%$ of people aged 70 years and older [3]. In China, the prevalence of MCI is $20.8 \%$ [4]. MCI has been attributed to numerous etiologies, such as Alzheimer's disease $(\mathrm{AD})$ and psychiatric disorders $[5,6]$. More than $40 \%$ of patients with MCI could revert to normal function, and $10-15 \%$ could progress to AD [7]. Additionally, diabetes and advanced age are high-risk factors for the progression of $\mathrm{MCI}$ to $\mathrm{AD}$, and women are more risk-prone $[8,9]$.
Several studies have shown that a higher serum homocysteine (Hcy) concentration increases the risk of cognitive function impairment, such as $\mathrm{AD}$, vascular dementia, and Parkinson's disease [10-15]. Serum Hcy levels and folate levels were related to cognitive performance, even in elderly subjects without dementia [16]. After the examination of seven polymorphisms of genes involved in Hcy metabolism, it was reported that only methylenetetrahydrofolate reductase (MTHFR) C677T (rs1801133) gene polymorphisms were associated with Hcy concentration [17].

MTHFR, the key enzyme of folate and Hcy metabolism [18], catalyzes the reduction of methylenetetrahydrofolate to methyltetrahydrofolate [18], which is involved in the methylation of Hcy to generate methionine to maintain the 


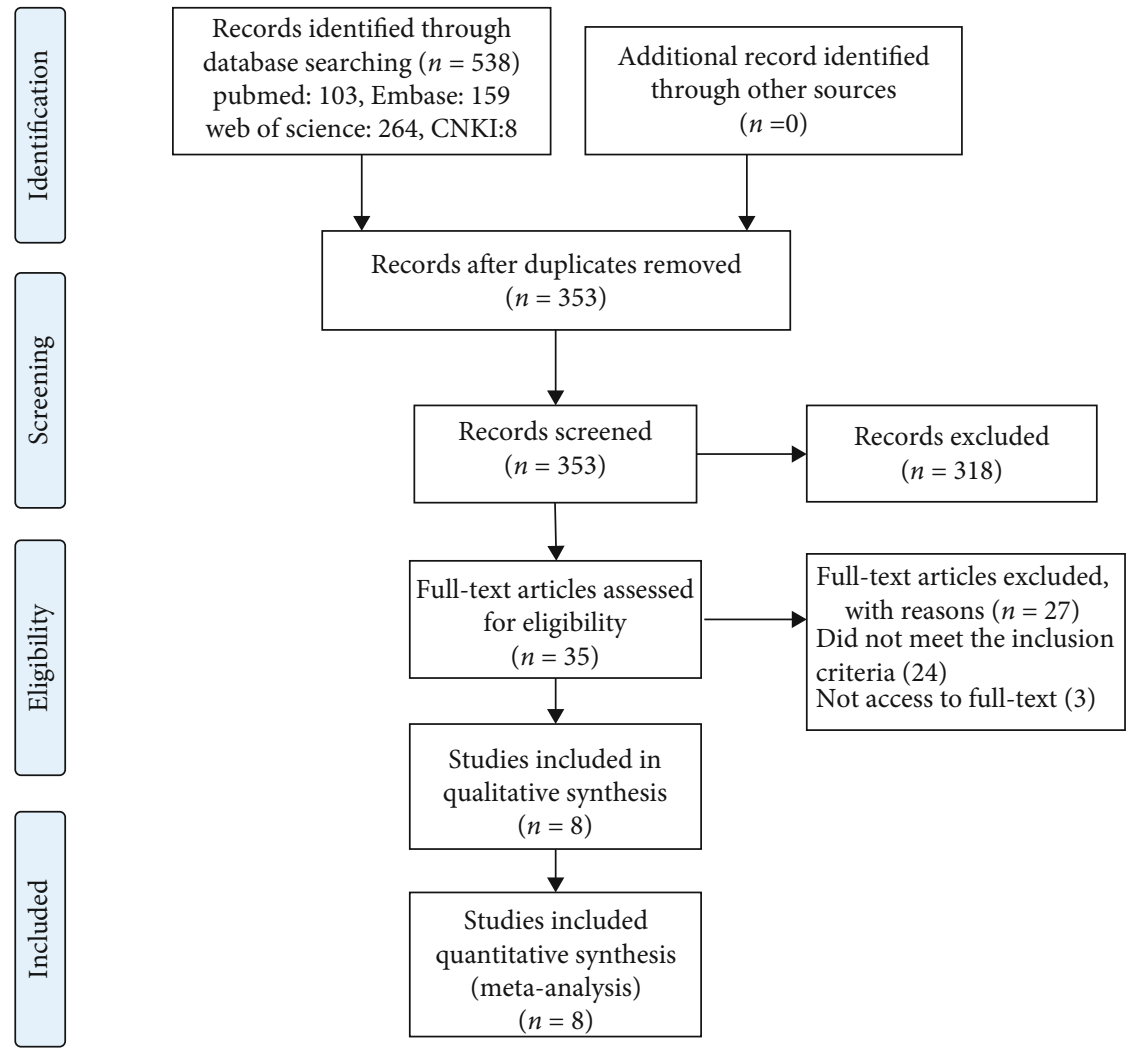

FIgURE 1: PRISMA flow diagram of the literature retrieval and selection process. CNKI: China National Knowledge Infrastructure.

serum Hcy concentration [19]. Variants in the MTHFR gene, where the cytosine is replaced by thymidine $(\mathrm{C} \rightarrow \mathrm{T})$ at nucleotide position 677 [20], are associated with elevated Hcy concentrations [21]. Severe MTHFR deficiency, a rare inherited disorder, can result in severe cognitive impairment [22]. Recently, a study found that omega-3 polyunsaturated fatty acids could predict cognitive impairment in those carrying the $\mathrm{T}$ variant after being stratified by MTHFR C677T (rs1801133) polymorphic allele carriage [23]. The TT genotype is associated with higher Hcy concentrations compared with the CC or CT genotypes, which means that individuals with the TT genotype who are exposed to higher serum Hcy concentrations for life should have a higher cognitive impairment than CC and CT individuals $[23,24]$. Participants with the TT genotype have been reported with $46 \%$ greater odds of cognitive impairment than those with the wild CC genotype $[25,26]$.

Extensive studies have reported that there was an association between MTHFR C677T (rs1801133) gene mutations and $\mathrm{AD}$ susceptibility [12, 27-29]. Several studies have reported relationships between MTHFR C677T (rs1801133) gene polymorphisms and the risk of MCI [30-32], the symptomatic predementia stage of AD; however, the results are inconsistent. Considering these previous contradictory results, this meta-analysis was conducted to evaluate the contribution of MTHFR C677T (rs1801133) gene polymorphisms to MCI susceptibility with greater precision.

\section{Materials and Methods}

2.1. Search Strategy. The systematic review and metaanalysis adhered to the Preferred Reporting Items for Systematic Reviews and Meta-Analyses (PRISMA) guidelines [33]. We performed a meta-analysis to determine the association between MTHFR C677T (rs1801133) polymorphisms and MCI susceptibility. PubMed, Embase, Web of Science, and China National Knowledge Infrastructure databases were searched from their inception to March 21, 2021, by two reviewers independently. The following search term combinations were used: (MTHFR or C677T or homocysteine) and (polymorphism or variant or mutation or SNP) and (cognitive or cognition), which were adjusted based on the characteristics of the database (Supplementary Table 1). A literature search was performed without restriction to region and publication types, and publication languages were restricted to either English or Chinese.

2.2. Inclusion and Exclusion Criteria. Studies included in the meta-analysis met the following criteria: (1) patients with MCI, (2) MTHFR C677T (rs1801133) gene polymorphism as the exposure factor, (3) control group individuals with normal cognitive function, and (4) case-control study design or cohort study design. The exclusion criteria were as follows: (1) repeated publication, (2) full text unavailable, and (3) genotype distributions unavailable for both cases and controls to calculate odds ratio (ORs) or $95 \%$ confidence intervals (CIs). 
TABLE 1: Study characteristics.

\begin{tabular}{|c|c|c|c|c|c|c|c|c|c|c|c|}
\hline \multirow[b]{2}{*}{ Author } & \multirow[b]{2}{*}{ Year } & \multirow[b]{2}{*}{ Country } & \multirow[b]{2}{*}{ Study type } & \multirow[b]{2}{*}{ Ethnicity } & \multicolumn{2}{|c|}{ Age $(y)$, mean \pm sd } & \multicolumn{2}{|c|}{ Gender } & \multicolumn{2}{|c|}{ Sample size } & \multirow[b]{2}{*}{ NOS } \\
\hline & & & & & MCI & Control & $\begin{array}{c}\text { MCI } \\
\text { Female } \\
(\%)\end{array}$ & $\begin{array}{c}\text { Control } \\
\text { Female } \\
(\%)\end{array}$ & MCI & Control & \\
\hline $\begin{array}{l}\text { Roussotte } \\
{[36]}\end{array}$ & 2017 & Turkey & Cohort study & Caucasian & $75.14 \pm 7.22$ & $76.15 \pm 4.98$ & $\begin{array}{c}128 \\
(35.65)\end{array}$ & $94(45.63)$ & 359 & 206 & 7 \\
\hline Luo [37] & 2015 & China & $\begin{array}{c}\text { Case-control } \\
\text { study }\end{array}$ & Caucasian & $64.32 \pm 6.42$ & $64.41 \pm 6.46$ & $55(42.64)$ & $55(42.31)$ & 129 & 131 & 7 \\
\hline Kaur [38] & 2018 & India & Cohort study & Asian & $52.66 \pm 10.23$ & $51.75 \pm 10.48$ & $\begin{array}{c}192 \\
(69.57)\end{array}$ & $81(27.18)$ & 263 & 276 & 5 \\
\hline El-Batch [39] & 2010 & Turkey & $\begin{array}{c}\text { Case-control } \\
\text { study }\end{array}$ & Caucasian & $62.86 \pm 6.97$ & $60.25 \pm 4.98$ & $13(46.43)$ & $15(75.00)$ & 28 & 20 & 7 \\
\hline Zou [40] & 2016 & China & $\begin{array}{l}\text { Case-control } \\
\text { study }\end{array}$ & Caucasian & $65.76 \pm 7.6$ & $64.44 \pm 6.2$ & $55(44.35)$ & $54(43.55)$ & 124 & 124 & 6 \\
\hline Xing [41] & 2019 & China & $\begin{array}{c}\text { Case-control } \\
\text { study }\end{array}$ & Asian & $65.46 \pm 5.89$ & $65.37 \pm 6.98$ & $23(46.00)$ & $24(40.00)$ & 50 & 60 & 6 \\
\hline Wang [42] & 2019 & China & $\begin{array}{c}\text { Case-control } \\
\text { study }\end{array}$ & Asian & $56.6 \pm 6.1$ & $55.8 \pm 6.2$ & $73(44.24)$ & $58(51.79)$ & 165 & 112 & 6 \\
\hline $\operatorname{Li}[43]$ & 2017 & China & $\begin{array}{c}\text { Case-control } \\
\text { study }\end{array}$ & Asian & $73.51 \pm 5.17$ & $69.02 \pm 5.4$ & $36(55.38)$ & $33(52.38)$ & 65 & 63 & 6 \\
\hline
\end{tabular}

MCI: mild cognitive impairment; NOS: Newcastle-Ottawa scale.

TABLE 2: Genotype frequency of MTHFR C677T gene polymorphisms in MCI patients and the control group.

\begin{tabular}{|c|c|c|c|c|c|c|c|c|c|c|c|}
\hline \multirow{3}{*}{ Author (year) } & \multicolumn{10}{|c|}{ Genotype } & \multirow{3}{*}{ HWE } \\
\hline & \multicolumn{6}{|c|}{ MCI patients } & \multicolumn{4}{|c|}{ Control } & \\
\hline & $\mathrm{C}$ & $\mathrm{T}$ & $\mathrm{CC}$ & $\mathrm{CT}$ & TT & $\mathrm{C}$ & $\mathrm{T}$ & CC & CT & $\mathrm{TT}$ & \\
\hline Roussotte (2017) [36] & 455 & 263 & 149 & 157 & 53 & 264 & 148 & 84 & 96 & 26 & $p>0.05$ \\
\hline Luo (2015) [37] & 175 & 83 & 62 & 51 & 16 & 190 & 72 & 67 & 56 & 8 & $p>0.05$ \\
\hline Kaur (2018) [38] & 451 & 75 & 194 & 63 & 6 & 444 & 108 & 179 & 86 & 11 & $p>0.05$ \\
\hline El-Batch (2010) [39] & 36 & 20 & 11 & 14 & 3 & 33 & 7 & 14 & 5 & 1 & $p>0.05$ \\
\hline Zou (2016) [40] & 181 & 67 & 61 & 59 & 4 & 173 & 75 & 62 & 49 & 13 & $p>0.05$ \\
\hline Xing (2019) [41] & 46 & 54 & 15 & 16 & 19 & 83 & 37 & 32 & 19 & 9 & $p<0.05$ \\
\hline Wang (2019) [42] & 216 & 114 & 82 & 52 & 31 & 165 & 59 & 68 & 29 & 15 & $p<0.05$ \\
\hline Li (2017) [43] & 54 & 76 & 17 & 20 & 28 & 77 & 49 & 28 & 21 & 14 & $p<0.05$ \\
\hline
\end{tabular}

MCI: mild cognitive impairment; HWE: Hardy-Weinberg Equilibrium; OR: odds ratio.

2.3. Data Extraction and Quality Evaluation. The data extracted from the candidate studies included the first author, publication year, country, study type, ethnicity, age, sex, MTHFR polymorphisms, allele and genotype distribution, and sample size. "C" is used to indicate the wild-type allele while " $\mathrm{T}$ " indicates a mutant allele of singlenucleotide polymorphisms (SNP), respectively $(\mathrm{C}>\mathrm{T})$. We assessed the quality of eight retrospective studies, according to the Newcastle-Ottawa Scale (NOS), by examining three factors: patient selection, comparability of the study groups, and assessment of outcome [34]. A score of 0-9 (allocated as stars) was allocated to each study. Studies achieving seven or more stars were considered high quality [35], while studies with six stars or less were considered of moderate or low quality. Two reviewers conducted data extraction and literature quality evaluation independently. Any disagreements were resolved through discussion with a third investigator.
2.4. Statistical Analyses. All analyses were performed using Stata version 15.0 (Stata Corporation, College Station, TX, USA). Five separate analyses, the allelic model ( $\mathrm{T}$ vs. C), dominant model (CT+TT vs. CC), recessive model (TT vs. $\mathrm{CC}+\mathrm{CT}$ ), heterozygous model (CT vs. CC), and homozygous model (TT vs. CC), were conducted in this metaanalysis. Pooled ORs and 95\%, 95\% CIs were used to assess the association between C677T (rs1801133) gene mutations and MCI susceptibility. Chi-square and $I^{2}$ tests were used to examine the heterogeneity among the studies. A fixed effects model was adopted if heterogeneity was acceptable $\left(p>0.10, I^{2}<50 \%\right)$; otherwise, a random effects model was adopted. The pooled OR was assessed using the $Z$ test and defined $p$ value $<0.05$ as statistical significance. The chisquare test was used to determine the Hardy-Weinberg equilibrium (HWE) for the genotype frequencies, and $p<$ 0.05 was considered a significant imbalance. Sensitivity 


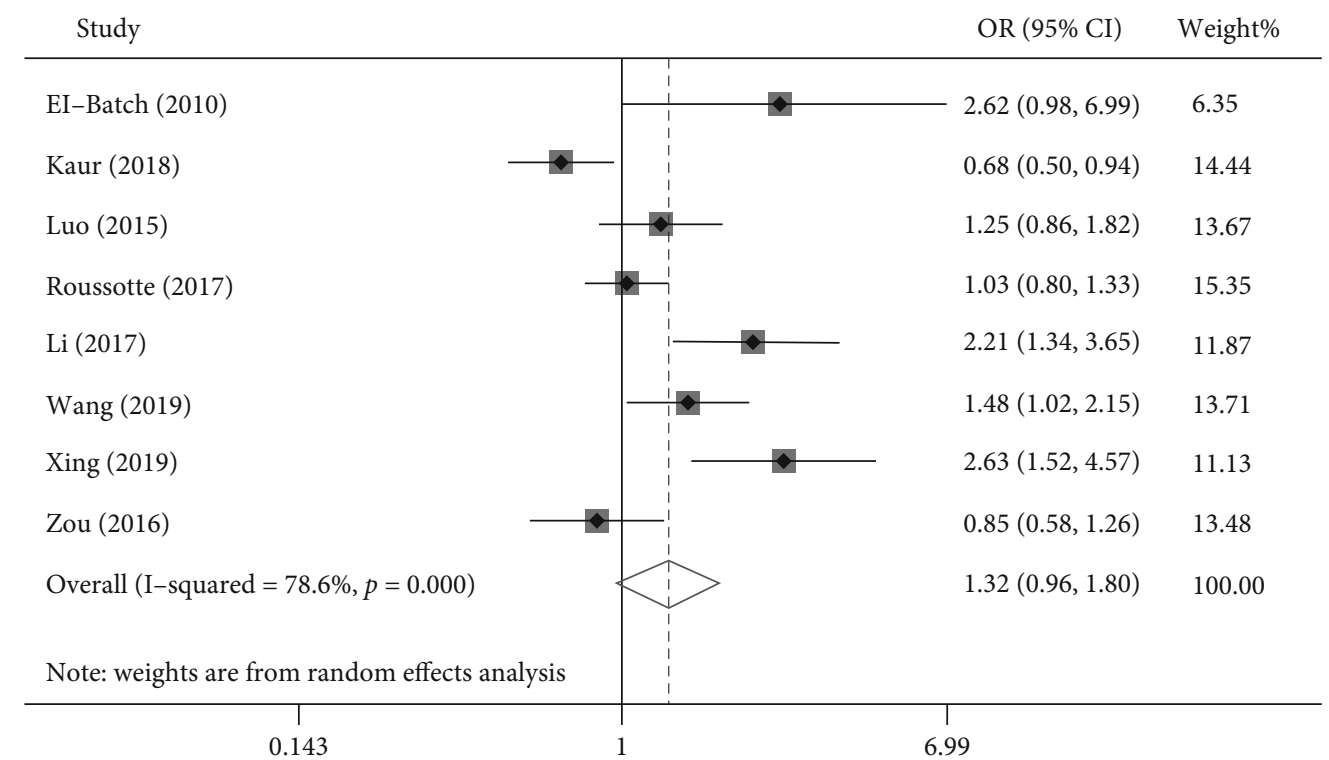

(a)

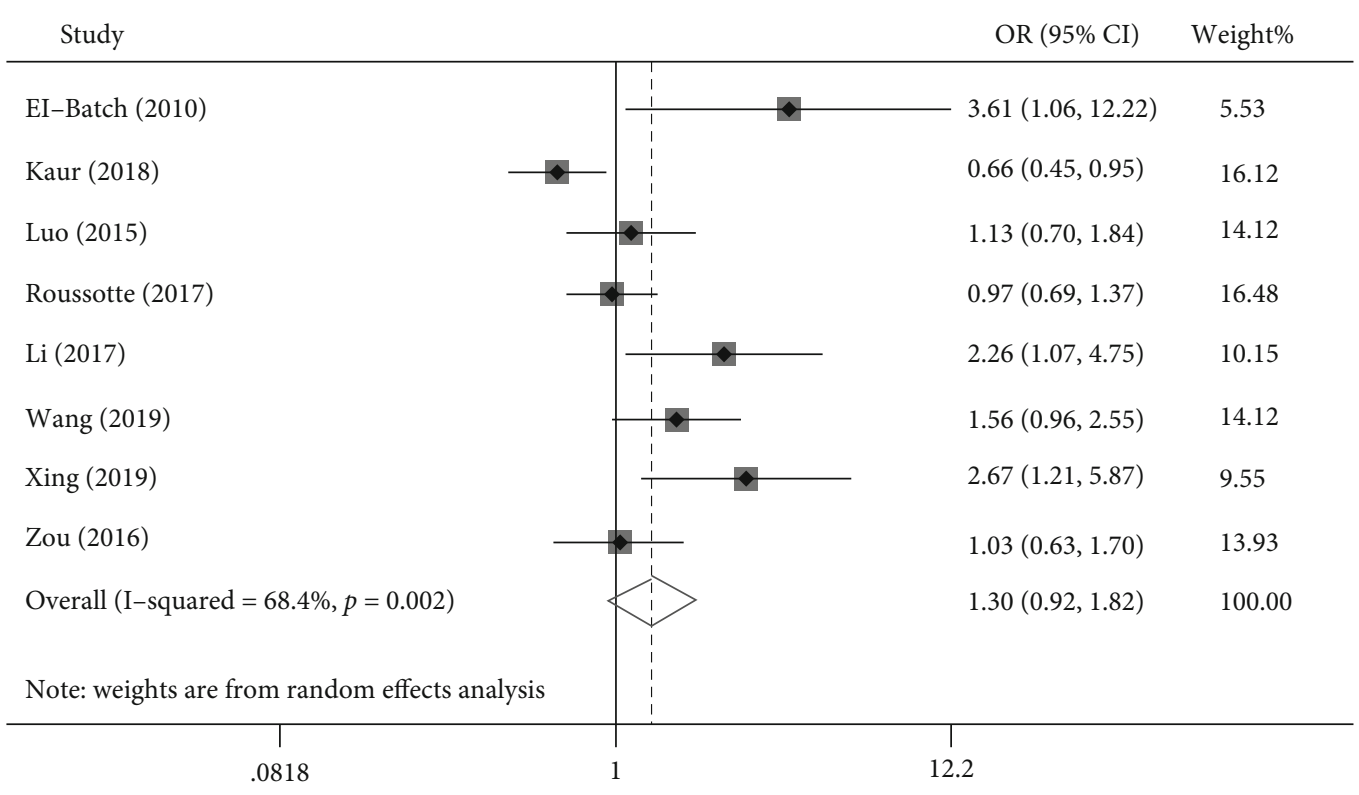

(b)

FIgUre 2: Continued. 


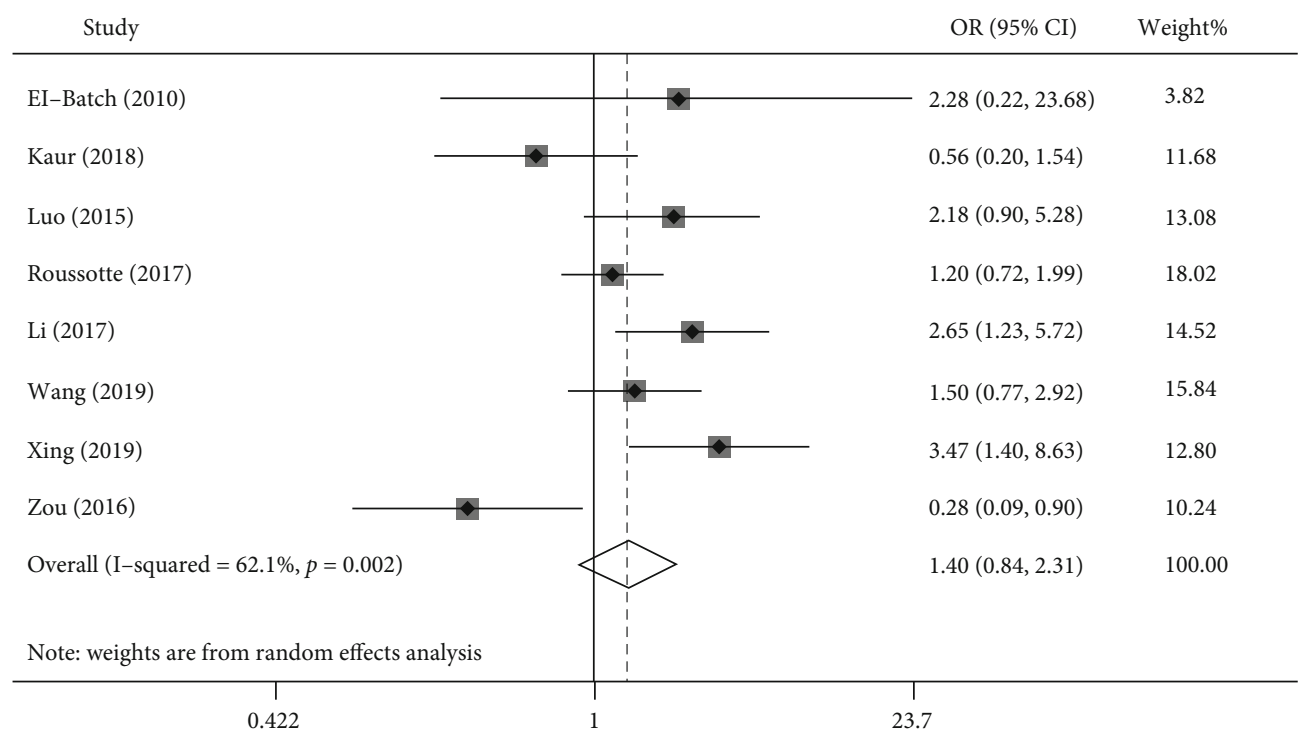

(c)

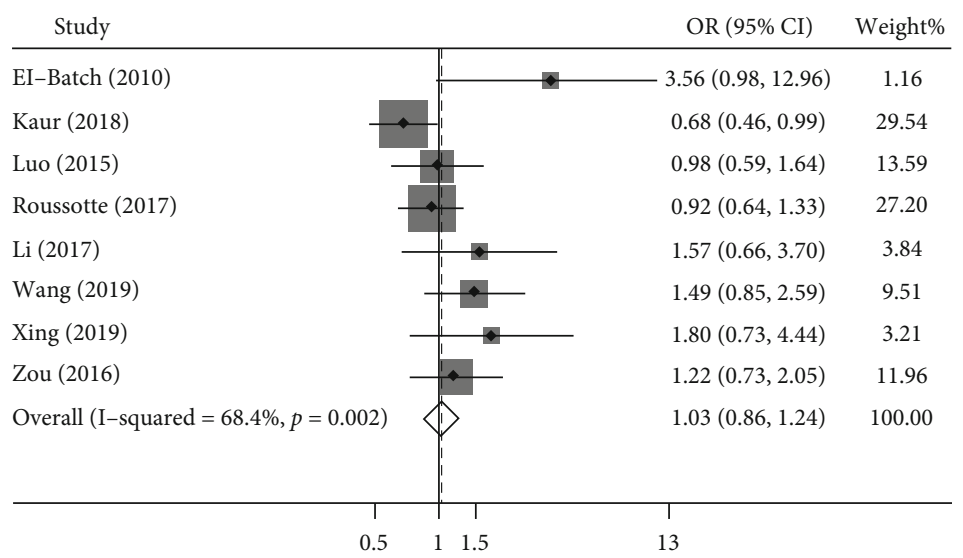

(d)

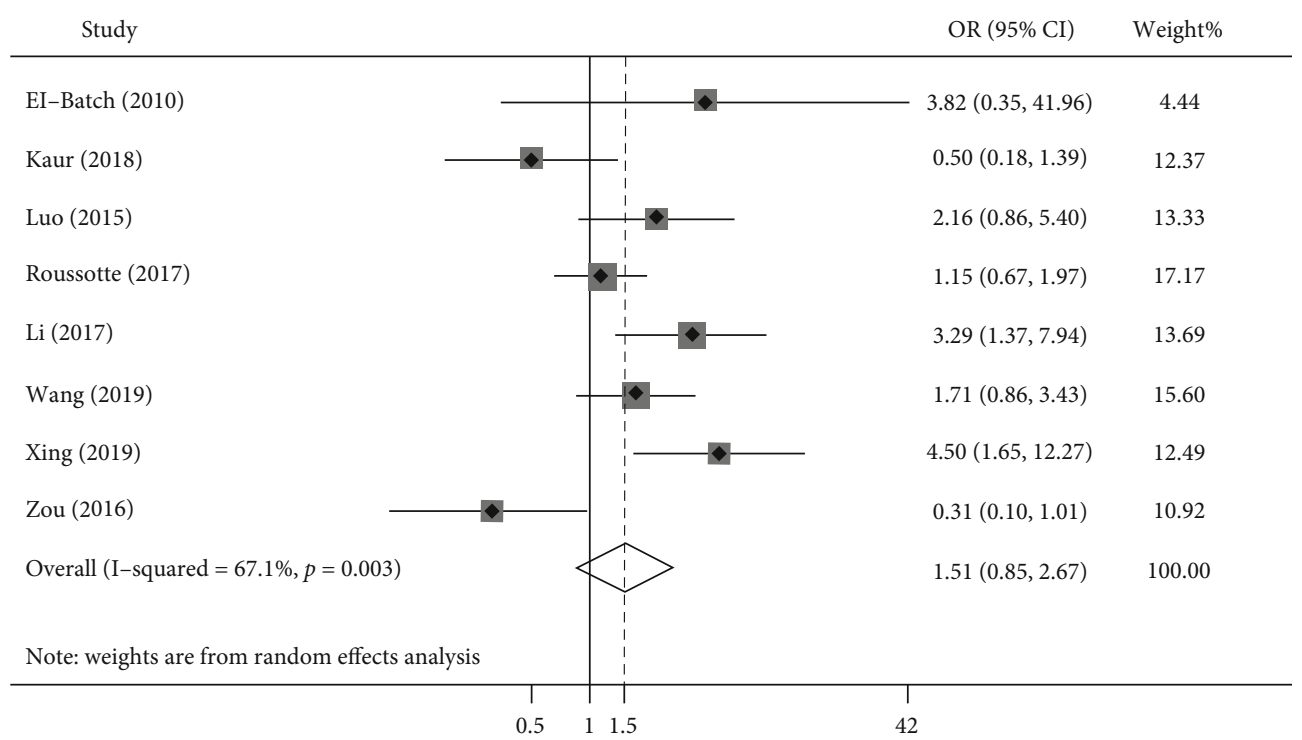

(e)

FIGURE 2: Forest plots of five gene models for the association between MTHFR C677T polymorphisms and mild cognitive impairment. (a) Allelic model (T vs. C), (b) dominant model (CT+TT vs.CC), (c) recessive model (TT vs. CC+CT), (d) heterozygous model (CT vs. CC), and (e) homozygous model (TT vs. CC). 
TABLE 3: Meta-analysis of the association between MTHFR C677T polymorphisms and MCI susceptibility and Egger's test.

\begin{tabular}{|c|c|c|c|c|c|c|c|c|c|}
\hline \multirow{2}{*}{ Comparison } & \multirow{2}{*}{$N$} & \multirow{2}{*}{ Model } & \multicolumn{4}{|c|}{ Pooled estimate value } & \multicolumn{2}{|c|}{ Heterogeneity } & \multirow{2}{*}{$p$ for Egger's test } \\
\hline & & & OR & $95 \%$ CI & $Z$ & $p$ & $I^{2}$ & $p$ & \\
\hline T vs. C & 8 & Random & 1.318 & $0.964-1.801$ & 1.73 & 0.084 & $78.6 \%$ & $\leq 0.001$ & 0.066 \\
\hline $\mathrm{CT}+\mathrm{TT}$ vs. CC & 8 & Random & 1.296 & $0.925-1.817$ & 1.51 & 0.132 & $68.4 \%$ & 0.002 & 0.006 \\
\hline TT vs. $\mathrm{CC}+\mathrm{CT}$ & 8 & Random & 1.397 & $0.845-2.312$ & 1.30 & 0.193 & $62.1 \%$ & 0.010 & 0.859 \\
\hline CT vs. CC & 8 & Fixed & 1.031 & $0.855-1.243$ & 0.32 & 0.749 & $46.3 \%$ & 0.071 & 0.005 \\
\hline TT vs. CC & 8 & Random & 1.506 & $0.850-2.667$ & 1.40 & 0.160 & $67.1 \%$ & 0.003 & 0.859 \\
\hline
\end{tabular}

$N$ : number of cases; OR: odds ratio; $95 \%$ CI: 95\% confidence intervals; $Z$ : $Z$ test; $p$ : $p$ value; $I^{2}: I^{2}$ test.

analysis for this meta-analysis was conducted by sequentially omitting one study at a time to evaluate the stability of the results. We performed subgroup analysis by stratification by ethnicity (Asian and Caucasian). To evaluate any potential publication bias, the funnel plot and Egger's linear regression tests were adopted, and publication bias was defined as a $p$ value of $<0.05$.

\section{Results}

3.1. Study Selection. A total of 538 potentially relevant studies were retrieved from the four databases. After 185 duplicates and 318 studies according to titles and abstracts were eliminated, the full texts of 35 articles were examined in detail; only eight papers [36-43] met all inclusion criteria, including four English [36-39] and four Chinese [40-43] articles. The detailed search process is illustrated in Figure 1.

3.2. Study Characteristics and Quality Evaluation. Eight studies were included in the present meta-analysis, involving 1,183 patients with MCI and 992 controls. Table 1 presents the study characteristics. Of these, six were case-control studies, and two were cohort studies. Among them, participants in four studies were Asian, and those in the remaining four studies were Caucasian. The mean age of all participants ranged from 51.75 to 76.15 years. Of the eight nonrandomized studies, three studies were classified as high-quality, while five studies were classified as moderate quality. The evidence included in this meta-analysis was considered of moderate quality owing to an average NOS score of 6.25. The distributions of MTHFR C677T (rs1801133) genotypes and allele frequencies in MCI cases and controls are shown in Table 2. The results of the HWE test for the distribution of genotypes in the control population are also displayed in Table 2; these were not in HWE for three studies.

3.3. Meta-Analysis of the Association between MTHFR C677T (rs1801133) Polymorphisms and MCI Susceptibility. The $I^{2}$ was less than $50 \%$ in the heterozygous model; therefore, a fixed effects model was adopted. Random effects models were adopted in other gene models because of significant heterogeneity $\left(I^{2}>50 \%\right)$. Forest plots of pooled ORs with the corresponding 95\% CIs are shown in Figure 2. The pooled results suggested that the MTHFR C677T (rs1801133) polymorphism was not significantly associated with the risk of MCI in any of the genetic frameworks, i.e., the allelic model (OR, 1.318; 95\% CI, 0.964-1.801; $p=$
TABLE 4: Subgroup analysis by ethnicity associated with MTHFR C677T gene polymorphisms and MCI susceptibility.

\begin{tabular}{lccc}
\hline Ethnicity & & Asian & Caucasian \\
\hline$N$ & T vs. C & 4 & 4 \\
OR & & $0.81-2.84$ & $0.84-1.44$ \\
$95 \%$ CI & & 4 & 4 \\
$N$ & CT+TT vs. CC & 1.49 & 1.11 \\
OR & & $0.75-2.97$ & $0.82-1.50$ \\
$95 \%$ CI & & 4 & 4 \\
$N$ & TT vs. CC+CT & 1.72 & 1.08 \\
OR & & $0.86-3.43$ & $0.47-2.46$ \\
$95 \%$ CI & & 4 & 4 \\
$N$ & CT vs. CC & 1.00 & 1.06 \\
OR & & $0.75-1.31$ & $0.82-1.37$ \\
$95 \%$ CI & & 4 & 4 \\
$N$ & TT vs. CC & 1.91 & 1.14 \\
OR & & $0.82-4.46$ & $0.50-2.59$ \\
$95 \%$ CI & & & \\
\hline
\end{tabular}

$N$ : number of cases; OR: odds ratio; 95\% CI: 95\% confidence interval; s.e.: standard error.

0.084), dominant model (OR, 1.296; 95\% CI, 0.925-1.817; $p=0.132$ ), recessive model (OR, 1.397; 95\% CI, 0.845$2.312 ; p=0.193$ ), heterozygous model (OR, 1.031; 95\% CI, $0.855-1.243 ; p=0.749$ ), or homozygous model (OR, 1.506; 95\% CI, 0.850-2.667; $p=0.160$ ) (Table 3).

3.4. Subgroup Analysis. A stratified subgroup analysis based on ethnicity was performed to investigate the exact consequences of the relationship between MTHFR C677T (rs1801133) gene polymorphisms and MCI susceptibility. Similar to the above results, no statistically significant association was observed in Caucasians or Asians under any genetic model between MCI risk and MTHFR C677T (rs1801133) genotype (Table 4).

3.5. Sensitivity Analysis. Sensitivity analysis was performed by omitting one study at a time to assess the robustness of the analysis to the results of individual studies. When single studies were removed one by one and the remaining studies were analyzed sequentially by meta-analysis, there was no significant change in the pooled ORs, indicating that the results were stabilized (Supplementary Figure 1). 


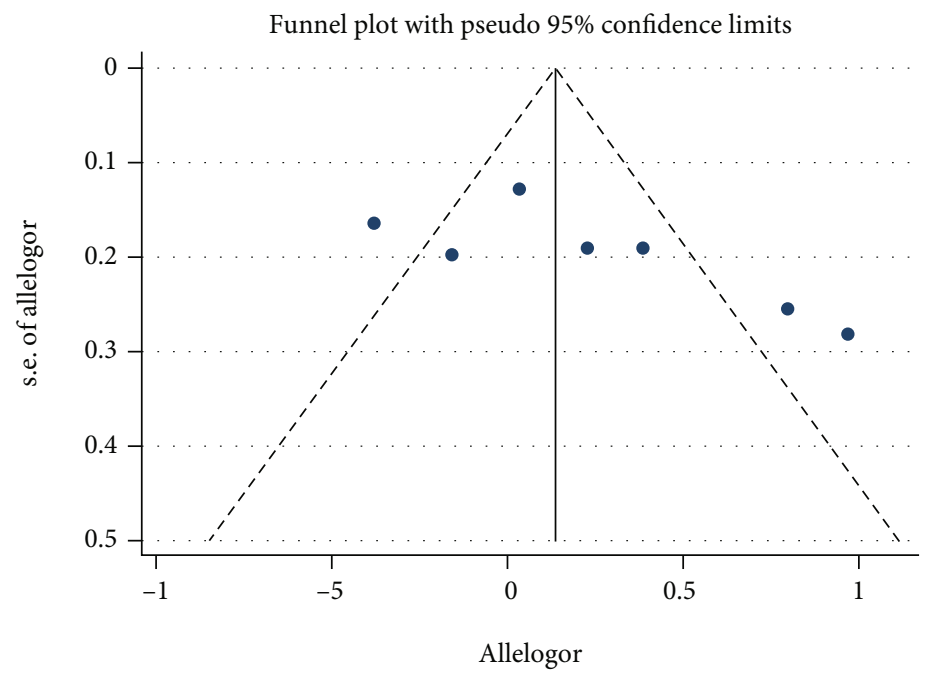

(a)

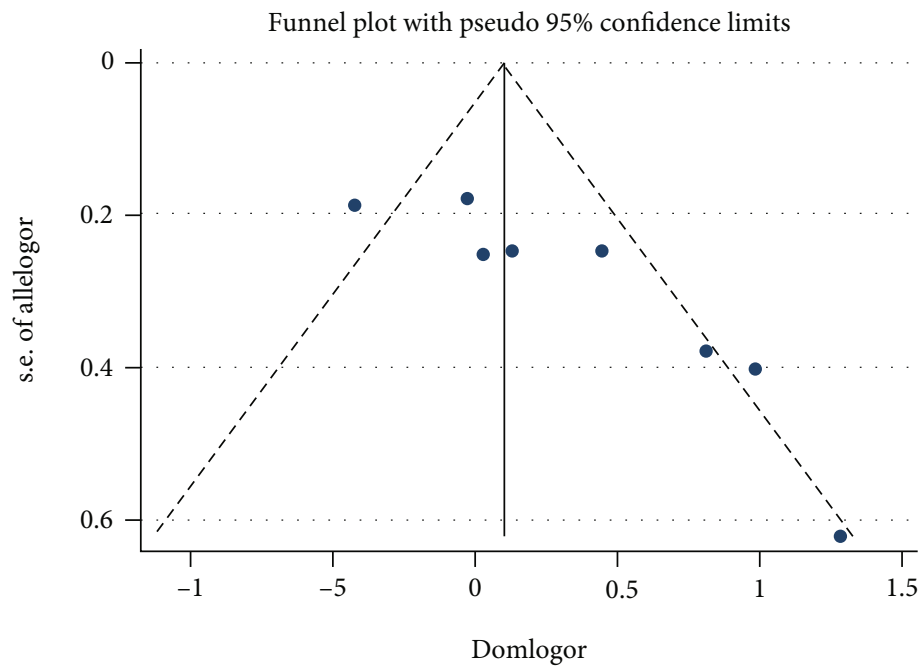

(b)

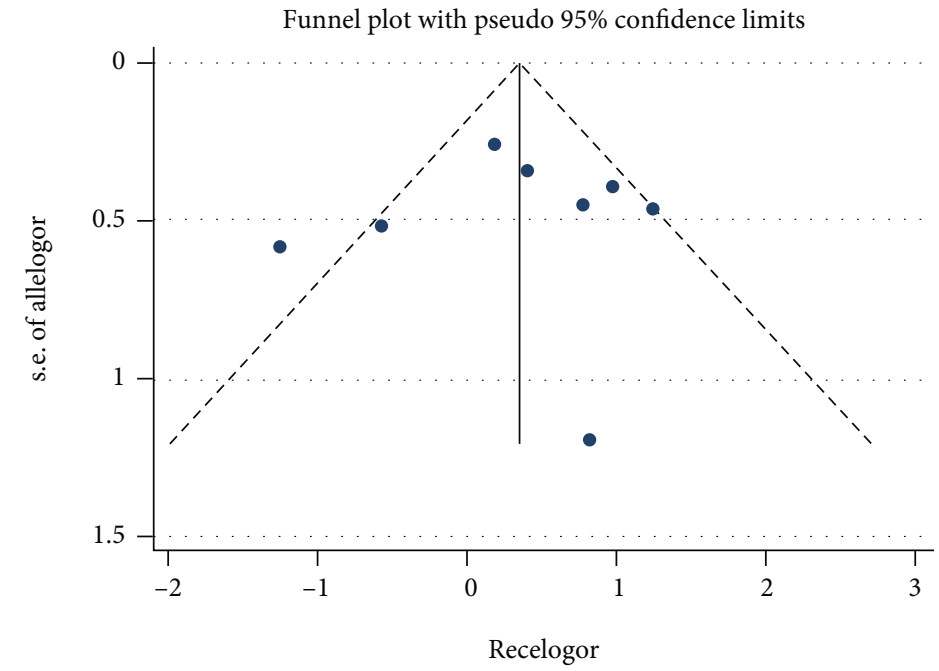

(c)

Figure 3: Continued. 


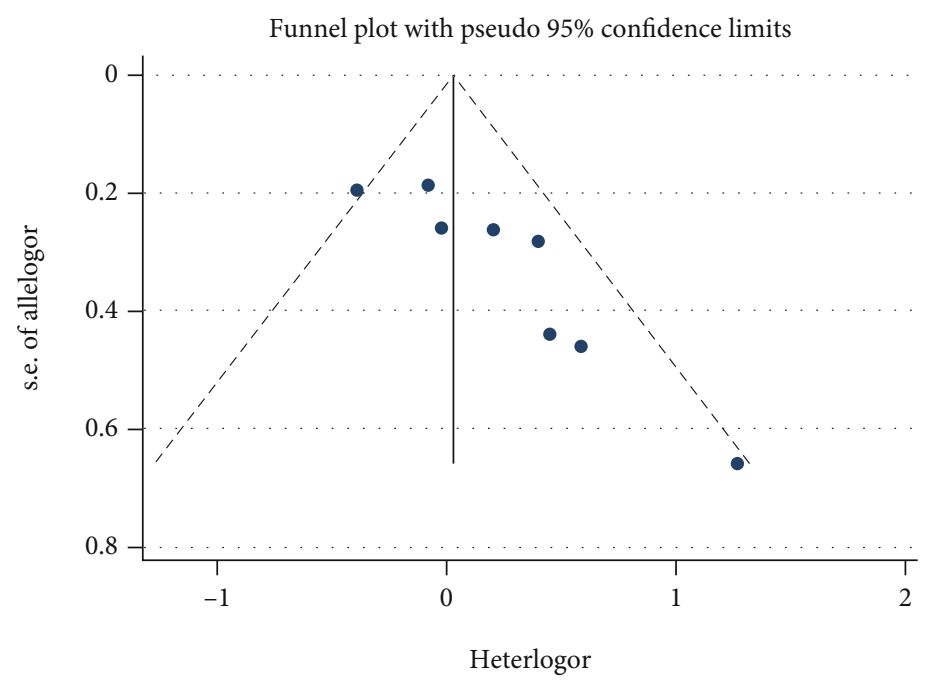

(d)

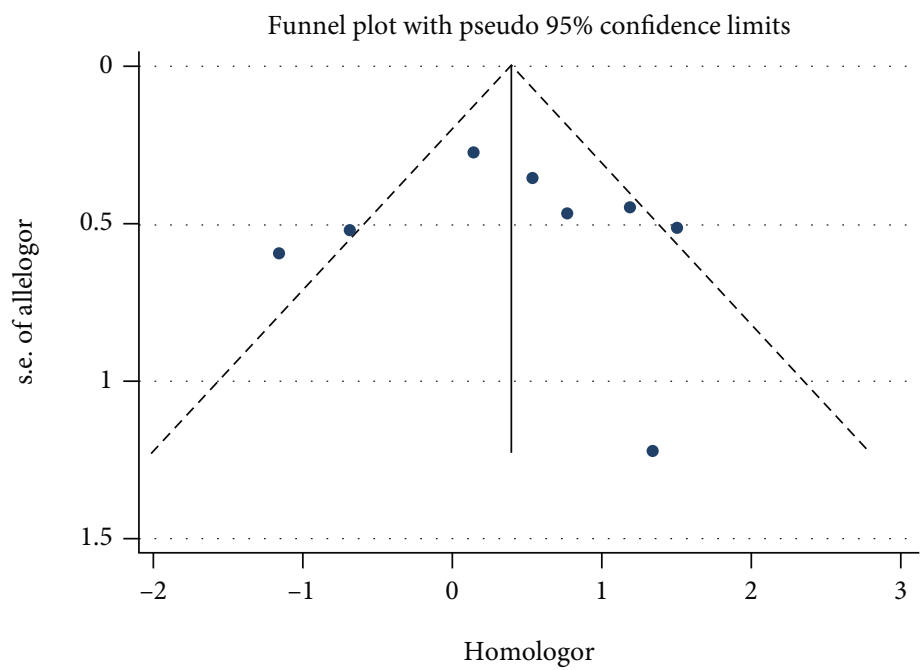

(e)

FIGURE 3: Funnel plot of five gene models for the association between MTHFR C677T polymorphisms and mild cognitive impairment. (a) Allelic model (T vs. C), (b) dominant model (CT+TT vs. CC), (c) recessive model (TT vs. CC+CT), (d) heterozygous model (CT vs. CC), and (e) homozygous model (TT vs. CC).

3.6. Publication Bias. The shapes of the funnel plots were roughly symmetrical in the allelic, recessive, and homozygous models (Figure 3). Egger's regression test indicated evidence of publication bias in the dominant $(p=0.006)$ and heterozygous ( $p=0.005)$ models, but no evidence of publication bias was found in the other gene models (Table 3 ).

\section{Discussion}

To our knowledge, this study represents the first meta-analysis of MTHFR C677T (rs1801133) gene polymorphisms and MCI susceptibility based on a broad range of studies involving 2,175 participants. In the current study, we did not find any statistically significant evidence that MTHFR C677T (rs1801133) gene variants can contribute to MCI susceptibility. No association between MTHFR C677T (rs1801133) gene polymorphisms and the risk of MCI was observed in the stratified analysis. The heterogeneity of the study was significant.
MTHFR, which depends on folate and vitamin B12, is a pivotal enzyme in one-carbon metabolism [44]. It has been reported that MTHFR C677T (rs1801133) gene variants result in lowered catalytic activity and are associated with elevated blood Hcy concentration [18, 45], which leads to a decline in cognitive function [30, 46-48]. It was found that adult cognition was associated with MTHFR gene polymorphisms and serum Hcy levels [49]. However, several subsequent studies have shown that MTHFR C677T (rs1801133) polymorphisms are not associated with individual changes in cognitive function $[23,50,51]$.

Previous reports have indicated that the MTHFR C677T (rs1801133) gene variant could contribute to AD susceptibility $[8,27]$. It has been reported that the one-carbon cyclederived methyl donor S-adenosylmethionine influenced the key gene expression, thereby affecting cognitive function $[52,53]$. First, the elevated blood Hcy concentration caused by MTHFR deficiency reduces the expression and 
methylation levels of Ser/Thr protein phosphatase 2A and leucine carboxylmethyltransferase 1 , resulting in tau dephosphorylation, which leads to the development of $\operatorname{AD}[46,54]$. Second, it was reported that a strong correlation exists between serum Hcy and plasma amyloid beta 40 (A $\beta 40)$ concentrations, which might result in AD [55]. Additionally, the TT genotype promotes an increase in plasma Hcy, which might favor intima media thickening in patients with cognitive impairment and cause cognitive function decline [56]. Finally, it was demonstrated that brain volume deficits were up to $5-12 \%$ in the MTHFR T allele group with MCI [57]. MCI was considered the symptomatic predementia stage; thus, these findings could explain the relationship between MTHFR C677T (rs1801133) gene polymorphisms and MCI.

SNPs are DNA sequence polymorphisms resulted by single-nucleotide mutations that occur at genomic levels, which might affect the expression or activity of the encoded protein and affect its function [58]. Genetic variants were fixed at conception and tended to be specific in their associations, which means that they did not change because of environmental factors [59-61]. It must be noted that the MTHFR C677T (rs1801133) gene polymorphisms of the three studies were not in HWE in control groups, which might have affected our findings. Therefore, the possible association is needed to verify by a representative sample.

In interpreting the results of the current research, a few limitations should be recognized. First, the results were highly heterogeneous, which may have distorted the meta-analysis. We considered the following possible sources of this heterogeneity: (i) different study types were pooled in our research, and (ii) inconsistent results might be limited by the ethnicity of the included population. We conducted a subgroup analysis based on ethnicity, but this did not affect the final result. Second, the sample size of our research was relatively small to investigate the association between MTHFR C677T (rs1801133) polymorphisms and MCI risk. Third, the literature search was restricted to articles published in Chinese and English, which might introduce publication and language bias. A limited number of electronic databases were investigated, and relevant studies might have been overlooked.

In conclusion, MTHFR C677T (rs1801133) gene polymorphisms were not associated with MCI susceptibility based on current studies. The TT genotype did not confer an increased risk of MCI compared to the CC and CT genotypes. However, considering the small sample size and limitations of the included research, further large-scale prospective studies and randomized controlled trials are needed to confirm our findings. In particular, future studies should take into account gene-gene and gene-environment interactions, as well as other confounding factors. We hope that our results will provide background data for future MCI research and will contribute to genetic marker screening.

\section{Data Availability}

PubMed, Embase, Web of Science, and China National Knowledge Infrastructure databases were searched from their inception to March 21, 2021.

\section{Conflicts of Interest}

The authors have no conflict of interest to report.

\section{Authors' Contributions}

J.S. and N.L. are responsible for the conceptualization. J.S. and N.L. are responsible for the methodology. J.S. is assigned to the software. X.J., M.Z., and L.M. did the validation. J.S. and N.L. did the formal analysis. J.S., X.J., and M.Z. performed the investigation. J.S. and N.L. acquired the resources. J.S. curated the data. J.S. did the writing and original draft preparation. H.L. and N.L. did the writing and review and editing. J.S. and X.J. did the visualization. L.M., H.P., and H.L. supervised the study. N.L. is assigned to the project administration. H.L. acquired funding. All authors have read and agreed to the published version of the manuscript. Jiahui Sun and Xuefan Jiang contributed equally to this work.

\section{Acknowledgments}

This research was funded by the National Natural Science Foundation of China (No. 81873350).

\section{Supplementary Materials}

Supplementary table 1 and figure 1 were appended in supplemental files. Supplementary table 1: search strategy. Supplementary figure 1: sensitivity analysis of five gene models for the association between MTHFR C677T polymorphisms and mild cognitive impairment. (a) Allelic model (T vs. C), (b) dominant model (CT+TT vs. CC), (c) recessive model (TT vs. CC+CT), (d) heterozygous model (CT vs. CC), and (e) homozygous model (TT vs. CC). (Supplementary Materials)

\section{References}

[1] R. C. Petersen, "Mild cognitive impairment as a diagnostic entity," Journal of Internal Medicine, vol. 256, no. 3, pp. 183194, 2004.

[2] V. Lissek and B. Suchan, "Preventing dementia? Interventional approaches in mild cognitive impairment," Neuroscience and Biobehavioral Reviews, vol. 122, pp. 143-164, 2021.

[3] B. L. Plassman, K. M. Langa, G. G. Fisher et al., "Prevalence of cognitive impairment without dementia in the United States," Annals of Internal Medicine, vol. 148, no. 6, pp. 427-434, 2008.

[4] J. Jia, A. Zhou, C. Wei et al., "The prevalence of mild cognitive impairment and its etiological subtypes in elderly Chinese," Alzheimers Dement, vol. 10, no. 4, pp. 439-447, 2014.

[5] A. Weston, C. Barton, J. Lesselyong, and K. Yaffe, "Functional deficits among patients with mild cognitive impairment," Alzheimers Dement, vol. 7, no. 6, pp. 611-614, 2011.

[6] K. M. Langa and D. A. Levine, "The diagnosis and management of mild cognitive impairment: a clinical review," JAMA, vol. 312, no. 23, pp. 2551-2561, 2014.

[7] L. Belcavello, D. Camporez, L. D. Almeida, R. L. Morelato, M. C. Batitucci, and F. de Paula, "Association of MTHFR and PICALM polymorphisms with Alzheimer's disease," Molecular Biology Reports, vol. 42, no. 3, pp. 611-616, 2015. 
[8] Q. Peng, X. Lao, X. Huang, X. Qin, S. Li, and Z. Zeng, “The MTHFR C677T polymorphism contributes to increased risk of Alzheimer's disease: evidence based on 40 case-control studies," Neuroscience Letters, vol. 586, pp. 36-42, 2015.

[9] O. J. Schiepers, M. P. van Boxtel, R. H. de Groot et al., "Genetic variation in folate metabolism is not associated with cognitive functioning or mood in healthy adults," Progress in NeuroPsychopharmacology \& Biological Psychiatry, vol. 35, no. 7, pp. 1682-1688, 2011.

[10] E. Setien-Suero, M. Suarez-Pinilla, P. Suarez-Pinilla, B. Crespo-Facorro, and R. Ayesa-Arriola, "Homocysteine and cognition: a systematic review of 111 studies," Neuroscience and Biobehavioral Reviews, vol. 69, pp. 280-298, 2016.

[11] K. Nilsson, L. Gustafson, and B. Hultberg, "Plasma homocysteine and cognition in elderly patients with dementia or other psychogeriatric diseases," Dementia and Geriatric Cognitive Disorders, vol. 30, no. 3, pp. 198-204, 2010.

[12] V. Rai, "Methylenetetrahydrofolate reductase (MTHFR) C677T polymorphism and Alzheimer disease risk: a metaanalysis," Molecular Neurobiology, vol. 54, no. 2, pp. 11731186, 2017.

[13] M. C. Bakeberg, A. Jefferson, M. Riley et al., "Elevated serum homocysteine levels have differential gender-specific associations with motor and cognitive states in Parkinson's disease," Parkinsons Disease, vol. 2019, 2019.

[14] R. C. M. Ho, M. W. L. Cheung, E. Fu et al., "Is high homocysteine level a risk factor for cognitive decline in elderly? A systematic review, meta-analysis, and meta-regression," American Journal of Geriatric Psychiatry, vol. 19, no. 7, pp. 607-617, 2011.

[15] R. Dwyer, O. A. Skrobot, J. Dwyer, M. Munafo, and P. G. Kehoe, "Using Alzgene-like approaches to investigate susceptibility genes for vascular cognitive impairment," Journal of Alzheimers Disease, vol. 34, no. 1, pp. 145-154, 2013.

[16] B. Hooshmand, A. Solomon, I. Kareholt et al., "Associations between serum homocysteine, holotranscobalamin, folate and cognition in the elderly: a longitudinal study," Journal of Internal Medicine, vol. 271, no. 2, pp. 204-212, 2012.

[17] L. M. de Lau, J. B. van Meurs, A. G. Uitterlinden et al., "Genetic variation in homocysteine metabolism, cognition, and white matter lesions," Neurobiology of Aging, vol. 31, no. 11, pp. 2020-2022, 2010.

[18] P. Goyette, J. S. Sumner, R. Milos et al., "Human methylenetetrahydrofolate reductase: isolation of cDNA, mapping and mutation identification," Nature Genetics, vol. 7, no. 2, pp. 195-200, 1994.

[19] G. S. Ducker and J. D. Rabinowitz, "One-carbon metabolism in health and disease," Cell Metabolism, vol. 25, no. 1, pp. 27-42, 2017.

[20] S. Hadj-Taieb, M. Feki, M. B. Hammami et al., "Plasma total homocysteine: usual values and main determinants in adults living in the great Tunis region," Clinical Laboratory, vol. 60, no. 6, pp. 897-902, 2014.

[21] N. Y. Chen, C. W. Liu, L. L. Du et al., "Enrichment of MTHFR $677 \mathrm{~T}$ in a Chinese long-lived cohort and its association with lipid modulation," Lipids in Health and Disease, vol. 13, no. 1, p. 104, 2014.

[22] H. Blessing, C. Weigel, J. Hammersen, W. Röschinger, and W. Rascher, "Normal development of a 7-month old boy after early start of treatment in severe methylenetetrahydrofolate reductase (MTHFR) deficiency identified by newborn screening," Monatsschrift fur Kinderheilkunde, vol. 167, p. 370, 2019.

[23] D. Moorthy, I. Peter, T. M. Scott et al., "Status of vitamins B-12 and B-6 but not of folate, homocysteine, and the methylenetetrahydrofolate reductase C677T polymorphism are associated with impaired cognition and depression in adults," The Journal of Nutrition, vol. 142, no. 8, pp. 1554-1560, 2012.

[24] H. J. Kim, I. W. Sohn, Y. S. Kim, and J. B. Jun, “The different relationship between homocysteine and uric acid levels with respect to the MTHFR C677T polymorphism according to gender in patients with cognitive impairment," Nutrients, vol. 12, 2020.

[25] A. H. Ford, L. Flicker, G. J. Hankey, P. Norman, F. M. Van Bockxmeer, and O. P. Almeida, "Homocysteine, methylenetetrahydrofolate reductase $\mathrm{C} 677 \mathrm{~T}$ polymorphism and cognitive impairment: the health in men study," Molecular Psychiatry, vol. 17, no. 5, pp. 559-566, 2012.

[26] A. H. Ford, L. Flicker, H. Alfonso et al., "Plasma homocysteine and MTHFRC677T polymorphism as risk factors for incident dementia," Journal of Neurology Neurosurgery and Psychiatry, vol. 83, no. 1, pp. 70-75, 2012.

[27] F. Zhou and S. Chen, "Hyperhomocysteinemia and risk of incident cognitive outcomes: an updated dose- response meta-analysis of prospective cohort studies," Ageing Research Reviews, vol. 51, pp. 55-66, 2019.

[28] T. Pi, B. Liu, and J. Shi, "Abnormal homocysteine metabolism: an insight of Alzheimer's disease from DNA methylation," Behavioural Neurology, vol. 2020, Article ID 8438602, 2020.

[29] L. Dayon, S. P. Guiraud, J. Corthésy et al., "One-carbon metabolism, cognitive impairment and CSF measures of Alzheimer pathology: homocysteine and beyond," Alzheimer's Research \& Therapy, vol. 9, no. 1, p. 43, 2017.

[30] J. Siuda, A. Gorzkowska, M. Patalong-Ogiewa et al., "From mild cognitive impairment to Alzheimer's disease - influence of homocysteine, vitamin B12 and folate on cognition over time: results from one-year follow-up," Neurologia i Neurochirurgia Polska, vol. 43, no. 4, pp. 321-329, 2009.

[31] O. P. Almeida, L. Flicker, N. T. Lautenschlager, P. Leedman, S. Vasikaran, and F. M. van Bockxmeer, "Contribution of the MTHFR gene to the causal pathway for depression, anxiety and cognitive impairment in later life," Neurobiology of Aging, vol. 26, no. 2, pp. 251-257, 2005.

[32] S. J. Tsai, C. J. Hong, H. L. Yeh et al., "Heterozygote advantage of the MTHFR C677T polymorphism on specific cognitive performance in elderly Chinese males without dementia," Dementia and Geriatric Cognitive Disorders, vol. 32, no. 3, pp. 159-163, 2011.

[33] A. C. Tricco, E. Lillie, W. Zarin et al., "PRISMA extension for scoping reviews (PRISMA-ScR): checklist and explanation," Annals of Internal Medicine, vol. 169, no. 7, pp. 467-473, 2018.

[34] A. Stang, "Critical evaluation of the Newcastle-Ottawa scale for the assessment of the quality of nonrandomized studies in meta-analyses," European Journal of Epidemiology, vol. 25, no. 9, pp. 603-605, 2010.

[35] D. A. Cook and D. A. Reed, "Appraising the quality of medical education research methods: the Medical Education Research Study Quality Instrument and the Newcastle-Ottawa ScaleEducation," Academic Medicine, vol. 90, no. 8, pp. 10671076, 2015.

[36] F. F. Roussotte, X. Hua, K. L. Narr, G. W. Small, and P. M. Thompson, "The C677T variant in_MTHFR_ modulates 
associations between brain integrity, mood, and cognitive functioning in old age," Biological Psychiatry: Cognitive Neuroscience and Neuroimaging, vol. 2, no. 3, pp. 280-288, 2017.

[37] M. Luo, H. Ji, X. Zhou, J. Liang, and T. Zou, "Correlation of homocysteine metabolic enzymes gene polymorphism and mild cognitive impairment in the Xinjiang Uygur population," Medical Science Monitor, vol. 21, pp. 326-332, 2015.

[38] G. Kaur, R. Gaur, S. Yadav, and K. N. Saraswathy, "Association of vitamin B12 mediated hyperhomocysteinemia and methylenetetrafolate reductase (C677T) gene polymorphism with cognitive impairment: a population based study from North India," Psychiatry Research, vol. 270, pp. 123-125, 2018.

[39] M. El-Batch, M. A. Eissa, G. Farouk, and M. Attia, "N-Terminal pro-brain natriuretic peptide, homocysteine and methylenetetrahydrofolate reductase gene polymorphism in elderly depressed and mild cognitive impairment patients," Turkish Journal of Biochemistry, vol. 35, pp. 105-113, 2010.

[40] T. Zou, X. Zhou, L. Ma, L. Zhang, and M. Zhang, "The association between the polymorphism of VDR gene and MTHFR gene in mild cognitive impairment of Uygurs in Xinjiang China," Chinese Journal of Clinical Neurosciences, vol. 24, pp. 645-653, 2016.

[41] H. Xing, R. Lu, N. Liang, and J. Zhou, "Relationship between amnesic mild cognitive impairment, MTHFR C677T gene polymorphism and plasma homocysteine," Level Molecular Diagnosis \& Therapy, vol. 11, pp. 22-27, 2019.

[42] J. Wang, L. Du, Y. Zhao et al., "Correlation and mechanism between constitution in traditional Chinese medicine and mild cognitive impairment of patients with type 2 diabetes mellitus," Shandong Journal of Traditional Chinese Medicine, vol. 38, 2019.

[43] Y. Li, H. Zeng, L. Zhou, L. Ma, and X. Wang, "Relationships of C677T polymorphism of methylenetetrahydrofolate reductase and plasma homocysteine levels to amnestic mild cognitive impairment," Practical Clinical Medicine, vol. 18, 2017.

[44] A. S. Tibbetts and D. R. Appling, "Compartmentalization of mammalian folate-mediated one-carbon metabolism," Annual Review of Nutrition, vol. 30, no. 1, pp. 57-81, 2010.

[45] H. Refsum, E. Nurk, A. D. Smith et al., "The Hordaland homocysteine study: a community-based study of homocysteine, its determinants, and associations with disease," Journal of Nutrition, vol. 136, no. 6, pp. 1731S-1740S, 2006.

[46] C. B. Brady, J. M. Gaziano, R. A. Cxypoliski et al., "Homocysteine lowering and cognition in CKD: the veterans affairs homocysteine study," American Journal of Kidney Diseases, vol. 54, no. 3, pp. 440-449, 2009.

[47] J. S. Elkins, S. C. Johnston, E. Ziv, D. Kado, J. A. Cauley, and K. Yaffe, "Methylenetetrahydrofolate reductase C677T polymorphism and cognitive function in older women," American Journal of Epidemiology, vol. 166, no. 6, pp. 672-678, 2007.

[48] T. Bottiglieri, L. Parnetti, E. Arning et al., "Plasma total homocysteine levels and the C677T mutation in the methylenetetrahydrofolate reductase (MTHFR) gene: a study in an Italian population with dementia," Mechanisms of Ageing and Development, vol. 122, no. 16, pp. 2013-2023, 2001.

[49] C. Cai, R. Xiao, N. Van Halm-Lutterodt et al., "Association of MTHFR, SLC19A1 genetic polymorphism, serum folate, vitamin B-12 and Hcy status with cognitive functions in Chinese adults," Nutrients, vol. 8, no. 10, p. 665, 2016.

[50] L. Polito, T. E. Poloni, R. Vaccaro et al., "High homocysteine and epistasis between_MTHFR_ and_APOE_ : association with cognitive performance in the elderly," Experimental Gerontology, vol. 76, pp. 9-16, 2016.

[51] O. J. Schiepers, M. P. van Boxtel, S. E. Harris et al., "MTHFR polymorphisms and cognitive ageing in the ninth decade: the Lothian Birth Cohort 1921," Genes, Brain, and Behavior, vol. 10, no. 3, pp. 354-364, 2011.

[52] C. Sugden, "One-carbon metabolism in psychiatric illness," Nutrition Research Reviews, vol. 19, no. 1, pp. 117-136, 2006.

[53] X. Lan, M. S. Field, and P. J. Stover, "Cell cycle regulation of folate-mediated one-carbon metabolism," Wiley Interdisciplinary Reviews. Systems Biology and Medicine, vol. 10, no. 6, 2018.

[54] M. Bednarska-Makaruk, A. Graban, A. Sobczynska-Malefora et al., "Homocysteine metabolism and the associations of global DNA methylation with selected gene polymorphisms and nutritional factors in patients with dementia," Experimental Gerontology, vol. 81, pp. 83-91, 2016.

[55] A. Viswanathan, S. Raj, S. M. Greenberg et al., "Plasma Abeta, homocysteine, and cognition: the Vitamin Intervention for Stroke Prevention (VISP) trial," Neurology, vol. 72, no. 3, pp. 268-272, 2009.

[56] G. Gorgone, F. Ursini, C. Altamura et al., "Hyperhomocysteinemia, intima-media thickness and C677T MTHFR gene polymorphism: a correlation study in patients with cognitive impairment," Atherosclerosis, vol. 206, no. 1, pp. 309-313, 2009.

[57] P. Rajagopalan, N. Jahanshad, J. L. Stein et al., "Common folate gene variant,_MTHFR_C677T, is associated with brain structure in two independent cohorts of people with mild cognitive impairment," NeuroImage: Clinical, vol. 1, no. 1, pp. 179-187, 2012.

[58] J. Zhao, W. Li, D. Zhu et al., "Association of single nucleotide polymorphisms in MTHFR and ABCG2 with the different efficacy of first-line chemotherapy in metastatic colorectal cancer," Medical Oncology, vol. 31, no. 1, p. 802, 2014.

[59] S. Burgess, D. S. Small, and S. G. Thompson, "A review of instrumental variable estimators for Mendelian randomization," Statistical Methods in Medical Research, vol. 26, no. 5, pp. 2333-2355, 2017.

[60] R. C. Richmond, G. Hemani, K. Tilling, G. Davey Smith, and C. L. Relton, "Challenges and novel approaches for investigating molecular mediation," Human Molecular Genetics, vol. 25, no. R2, pp. R149-R156, 2016.

[61] H. Lu, P. F. Wu, W. Zhang, and X. Liao, "Circulating interleukins and risk of multiple sclerosis: a Mendelian randomization study," Frontiers in Immunology, vol. 12, p. 647588, 2021. 